Archives

$45 \mid 2010$

La preuve en histoire

\title{
Les archives du communisme roumain : sources historiques et preuves en justice
}

Le cas de l'Institut d'Investigation des Crimes du Communisme en

Roumanie (2005-2007)

Laurențiu Vlad

\section{(2) OpenEdition}

Journals

Édition électronique

URL : http://journals.openedition.org/ccrh/3569

DOI : $10.4000 /$ ccrh.3569

ISSN : 1760-7906

\section{Éditeur}

Centre de recherches historiques - EHESS

\section{Édition imprimée}

Date de publication : 25 avril 2010

Pagination : 195-200

ISSN : 0990-9141

\section{Référence électronique}

Laurențiu Vlad, « Les archives du communisme roumain : sources historiques et preuves en justice », Les Cahiers du Centre de Recherches Historiques [En ligne], 45 | 2010, mis en ligne le 10 janvier 2012, consulté le 20 avril 2019. URL : http://journals.openedition.org/ccrh/3569 ; DOI : 10.4000/ccrh.3569

Ce document a été généré automatiquement le 20 avril 2019.

Article L.111-1 du Code de la propriété intellectuelle. 


\section{Les archives du communisme roumain: sources historiques et preuves en justice}

Le cas de l'Institut d'Investigation des Crimes du Communisme en

Roumanie (2005-2007)

\section{Laurențiu Vlad}

\section{Juge et commissaire de police}

1 Vers la fin de la septième décennie du XVIII ${ }^{e}$ siècle, plus exactement en 1769 , dans son Traité des différentes sortes de preuves qui servent à établir la vérité de l'histoire, le jésuite Henri Griffet compare les historiens aux juges ${ }^{1}$. L'analogie d'Henri Griffet apparait plus tard dans les études historiographiques, un des plus récents exemples étant dans le volume de Carlo Ginzburg publié en 1991, Il giudice e lo storico. Il est bien connu que les juges tout comme les historiens utilisent des preuves pour établir le déroulement des événements et des faits du passé, autrement dit, des vérités :

Le métier des uns et des autres se fonde sur la possibilité de prouver, en fonction de règles déterminées que $\mathrm{X}$ a fait $\mathrm{Y} ; \mathrm{X}$ pouvant désigner indifféremment le protagoniste, éventuellement anonyme, d'un événement historique ou le sujet impliqué dans une procédure pénale; et $Y$ une action quelconque ${ }^{2}$.

Ceci est valable aujourd'hui aussi, même si, sous l'influence extraordinaire des études sur les « représentations », l'historien est plutôt tenté de voir dans les sources qu'il utilise les différentes manières de leur production et de leur construction, et non les preuves d'un procès de rétablissement d'une vérité ou de la description d'une réalité ${ }^{3}$.

2 Mais Carlo Ginzburg n'hésite pas à le dire, dans Mythes, emblèmes, traces. Morphologie et histoire 4 que le travail de l'historien est également celui d'un commissaire de police qui cherche sans cesse des preuves et qui les utilise pour reconstituer avec minutie des événements, des faits, des représentations ou des vérités. L'historien vu comme juge ou 
comme commissaire de police est évidemment plus proche du domaine spécifique, classique pourrait-on même dire, de l'utilisation de la preuve, à savoir la justice. Ce plaidoyer n'est plus nécessaire aujourd'hui, car, selon William Twining, qui cite Jeremy Bentham, «the field of evidence is no other than de field of knowledge ${ }^{5}$. D'autant plus que, poursuit William Twining, dans les conditions d'un intérêt croissant pour la récupération de la « mémoire » (et pour son institutionnalisation, nous allons ajouter), les historiens associent d'une manière naturelle la reconstruction du passé à l'imaginaire et à la preuve (« evidence $)^{6}{ }^{6}$.

3 Dans ce qui suit, nous présenterons un cas particulier dans lequel le document (d'archives ou de bibliothèques) peut avoir la valeur d'une source (disons preuve, témoignage) historique, mais aussi d'une preuve (disons source, témoignage) en justice. Il ne s'agit donc pas d'une association symbolique, basée strictement sur une manière spécifique de travail, du domaine historiographique et celui de la justice. Ce double aspect est illustré, dans notre opinion, par les objectifs et l'activité de l'Institut d'Investigation des Crimes du Communisme en Roumanie (IICCR).

\section{L'Institut d'investigation des crimes du communisme en Roumanie ${ }^{7}$}

4 L'Institut d'Investigation des Crimes du Communisme en Roumanie (IICCR), a été fondé sur décision du Gouvernement ${ }^{8}$ comme une institution dotée de personnalité juridique subordonnée au gouvernement et sous la direction du Premier ministre. Les objectifs principaux de l'Institut, selon les prévisions de la Décision du Gouvernement, sont, d'un côté, « l'investigation scientifique et l'identification des crimes, des abus et des atteintes aux droits de l'homme » qui ont eu lieu pendant le régime communiste de Roumanie et, de l'autre côté, de " saisir les institutions de droit dans les cas où des situations contraires à la loi sont découvertes" (art. 2). Pour atteindre ces objectifs, l'IICCR inventorie des documents, des témoignages et des données sur les activités de répression politique de l'État communiste qu'il donne ensuite à la Procurature, tout en les présentant au public et en en identifiant les responsables (art. 3). L'IICCR crée en même temps des bases de données documentaires sur les types de crimes et d'abus constatés pendant les investigations, sur la nomenklatura communiste et les employés de la Securitate, sur la législation en vigueur pendant la période du régime totalitaire etc. (art. 4) Il faut ajouter que l'activité de l'IICCR est coordonnée par un conseil composé de onze personnalités de la société civile ${ }^{9}$, nommées par le Premier ministre, qui élisent un comité directeur (exécutif) ${ }^{10}$ composé d'un président et de deux membres (art. 6).

Une année et demie après sa création, les autorités ont voulu rendre plus clairs, sans doute à la suggestion de la direction de l'Institut également, certains aspects de son activité. Par conséquent, l'article unique de la nouvelle Décision du Gouvernement ${ }^{11}$ définit plus clairement les activités d'édition, de musée, de recherche scientifique, de coopération avec les institutions non-gouverne-mentales, académiques ou universitaires du pays ou de l'étranger. La Décision du Gouvernement de 2007 élimine la prévision selon laquelle une durée limite de fonctionnement de l'Institut avait été établie et elle prévoit aussi la possibilité que l'Institut puisse, par ses experts ou des collaborateurs externes, entreprendre des activités pour «obtenir des dédommagements moraux et matériels pour les victimes du régime communiste et de l'ancienne Securitate ». De même, 
l'organigramme de l'IICCR a été élargie (de vingt-cinq à trente-cinq employés - jeunes historiens, politistes, sociologues etc.) - et le mandat du président de l'institution a été fixé à six ans, renouvelable une seule fois.

6 Il existe dans l'organigramme de l'IICCR une Direction Générale d'Investigations (DGI), qui a deux bureaux (compartiments): un bureau de documentation et un bureau de recherches dans les archives. Toute l'activité de l'institut est en fait concentrée dans cette Direction. Les experts du Bureau de Documentation étudient «les sources ouvertes» (la presse et autres publications) de l'époque du régime communiste et ils cherchent à identifier les personnes qui ont eu des fonctions de décision dans l'appareil de répression, tandis que le Bureau de Recherches dans les Archives s'occupe de l'identification et l'étude des documents internes des organes du parti communiste et de la Securitate sur les abus et les crimes, de même que de l'identification de ceux qui les ont ordonnés.

7 Les résultats des recherches de la DGI sont structurés sur deux plans. Le premier plan est l'utilisation du document historique comme preuve en justice. C'est le premier objectif de l'IICCR, signalé d'une manière explicite par la Décision du Gouvernement du 2005. Le second plan, formulé surtout par la Décision du Gouvernement du 2007, restitue au document sa propre caractéristique de source historiographique ou d'instrument éducatif pour la construction de la mémoire collective.

8 Les plaintes pénales font partie de la première catégorie. Jusqu'à ce jour, quatre plaintes ont été finalisées ayant comme fondement les documents d'archives ou les données des sources ouvertes. Il s'agit des plaintes pénales du 4 décembre 2006, sur le racolage des mineurs par la Securitate, du 22 mai 2007, sur les abus et les crimes de deux cents dix officiers du système pénitentiaire roumain pendant les années 1945-1989, du 9 août 2007, sur des faits, surtout des enquêtes abusives et des tortures commises par un officier supérieur du Ministère des Affaires Intérieures et du 7 novembre 2007, sur l'organisation d'activités terroristes. Outre ces démarches, il faut également mentionner une série d'autres investigations spéciales qui ne servent pas à déposer des plaintes pénales, mais sont stockées dans plusieurs bases de données ${ }^{12}$.

9 Les recherches proprement dites font partie de la seconde catégorie. Elles se sont matérialisées dans la publication d'études scientifiques ${ }^{13}$ dans l'organisation d'expositions ou de concours éducatifs (par exemple, le concours d'art graphique et d'essais pour des écoliers intitulé "Que signifie le communisme pour moi?», ou l'exposition itinérante "À travers les lignes: les échos des événements de 1956 en Roumanie »), dans des programmes d'études conçus avec des universités : par exemple, l'inauguration du Centre d'Études sur le Communisme et le Post-communisme en collaboration avec la Faculté d'Histoire de l'Université « Alexandru Ioan Cuza » de Iaşi, dont le premier projet est la création d'un programme de Master.

\section{Ni juge, ni commissaire de police}

10 L'analyse des données de la section précédente indique de toute évidence que l'IICCR est un organisme qui se propose de construire la mémoire actuelle du communisme autochtone. C'est ce que font en République Tchèque le Museum of Communism et le Czech office for the Documentation and the Investigation of the Crimes of Communism, ou en Hongrie la House of Terror et l'Institute for the History of the 1956 Hungarian Revolution, ou en Pologne l'Institute of National Remembrance. 
11 Comme toute initiative de ce genre, l'IICCR subit l'influence de nombreux impératifs contemporains ; Lucien Febvre et Jacques Le Goff ont brillamment illustré de tels aspects ${ }^{14}$. Dans ce qui suit, nous allons évoquer quelques-uns en ce qui concerne l'IICCR.

12 Ainsi, l'IICCR n'est pas simplement un espace de récupération et de conservation de la mémoire (un musée ou une démarche d'ordre éducatif). Il se manifeste également sur le plan du militantisme réparateur par la poursuite des coupables ayant commis des atrocités et des crimes pendant le régime communiste. Par conséquent, on peut identifier une tentative nette de délimitation de la simple condamnation globale et symbolique d'un régime abusif. L'IICCR est plutôt enclin à individualiser la culpabilité ; c'est la raison pour laquelle il mène une série d'investigations ponctuelles et met à la disposition des organes d'enquête pénale des données obtenues par les recherches dans les archives (y compris d'histoire orale) ou dans les bibliothèques. La philosophie dominante d'une telle démarche est le libéralisme classique, selon lequel toute culpabilité est individualisée, et le coupable doit se présenter devant un juge qui délibérera et condamnera en connaissance de cause. Cette philosophie n'est pas due au hasard, car tant le président (Marius Oprea) que le directeur général (Stejărel Olaru) de l'IICCR sont proches du Parti National Libéral ; le premier s'est présenté comme candidat pour la fonction de viceprésident de ce parti politique lors des dernières élections internes, tandis que le second est parmi les premiers sur la liste pour les élections euro-parlementaires de novembre 2007. Quoiqu'il en soit, l'IICCR ne peut pas se faire juge (peut-être seulement dans un tribunal moral), il ne peut être qu'un commissaire de police selon le schéma que nous avons indiqué au début de cette intervention.

13 Mais l'IICCR est également un espace de la recherche scientifique, les annuaires de 2006 et 2007, de même que les études individuelles publiées par ses employés le prouvent largement (7 volumes sous l'égide de l'Institut en 2007). Sauf qu'il a une série de concurrents redoutables, qui, honnêtement, sont inscrits sur le site officiel de l'IICCR parmi ses collaborateurs. Nous nous arrêtons à un seul, à savoir l'Institut pour l'Étude du Totalitarisme de l'Académie Roumaine, créé en 1993 et qui a déjà une production scientifique impressionnante: une revue déjà consacrée (Arhivele Totalitarismului [Les Archives du totalitarisme]), de nombreuses études et éditions de documents publiées par ses membres (environ 80 volumes depuis 1994). C'est la raison pour laquelle les chercheurs de l'establishment académique critiquent assez souvent les instituts créés plus récemment, qui, disent-ils, ne font pas preuve d'une véritable capacité de recherche scientifique sérieuse. C'est le point de vue exprimé publiquement, lors de l'anniversaire de sept décennies depuis la fondation de l'Institut d'Histoire "Nicolae Iorga " de l'Académie Roumaine, par son directeur, Ovidiu Cristea, sans qu'il en donne des noms ${ }^{15}$. À la même occasion, le président de la Roumanie, Traian Băsescu, en forte rivalité avec le premier ministre libéral, Călin Popescu-Tăriceanu, dont dépend l'IICCR, affirmait sans équivoque que dans certaines institutions on étudie le régime communiste sur des positions partisanes ${ }^{16}$. Par conséquent, il semble que l'IICCR a des inconvénients également dans le domaine historiographique. Sa position de commissaire de police semble également chanceler!

14 Pour conclure, nous disons que l'IICCR est l'expression d'une tension entre la tentation de se manifester d'une manière légitime, parfois d'une façon « impériale », dans le domaine récemment constitué de l'histoire politique roumaine contemporaine (l'étude du communisme autochtone), et l'intention de fournir des preuves, sur la base des sources historiques, pour les institutions ayant le droit d'ouvrir en justice des procès contre les 
anciens représentants, plus ou moins importants, du pouvoir communiste. Le besoin de légitimation de l'IICCR est double, car il est actif dans deux champs intellectuels distincts, ayant, pour chaque cas de figure, des positions ambigües. Dans le domaine historiographique, il n'a pas un statut d'autorité, tandis que dans le domaine de la gestion de la mémoire du communisme, fortement politisé en ce moment à Bucarest, l'utilisation de l'action de la société civile comme instance morale, n'est pas capable de lui donner une identité précise (voir à cet égard la concurrence avec la Commission Présidentielle pour l'Analyse de la Dictature Communiste de Roumanie ou le Conseil National pour l'Étude des Archives de la Securitate, les deux entités étant en même temps, selon le site officiel de l'IICCR ses partenaires). D'où le statut ambivalent de l'usage de la source (preuve) historique (juridique) par l'IICCR.

L'IICCR a fonctionné jusqu'au printemps 2010 quand, par une décision gouvernementale motivée par des raisons économiques, il a fusionné avec l'Institut National pour la Mémoire de l'Exil Roumain (INMER). On nomma une nouvelle direction: Vladimir Tismăneanu (Université de Maryland) et Ioan Stanomir (Université de Bucarest). Le premier avait auparavant été le chef de la Commission Présidentielle pour l'Analyse de la Dictature Communiste en Roumanie, le second, le responsable d'une autre commission constituée sous le patronage de Traian Băsescu, celle pour la révision de la Constitution...

\section{NOTES}

1. Carlo Ginzburg, Le juge et l'historien. Considérations en marge du procès Sofri, Paris, Éditions Verdier, 2007, p. 17. Ginzburg cite dans ce contexte Allen Johnson, The Historian and the Historical Evidence, New York, 1934 (première édition C. Scribner'sSons, 1926).

2. Ibid., p. 24. Mais aussi, Luigi Ferrajoli, Diritto e ragione. Teoria del garantissimo penale, Bari, Laterza, 1989, p. 32 : «Le procès est, pour ainsi dire, le seul cas d'« expérimentation historiographique ": on y fait jouer les sources de vivo, non seulement parce qu'elles sont recueillies directement, mais aussi parce qu'elles sont confrontées les unes aux autres, soumises à des examens croisés et incitées à reproduire, comme dans un psychodrame, l'affaire qui est jugée. »

3. Ibid., p. 22.

4. Carlo Ginzburg, Mythes, emblèmes, traces. Morphologie et histoire, Paris, Flammarion, 1989.

5. William Twining, «Evidence as a Multi-Disciplinary Subject ", dans Law, Probability and Risk, 2, p. 91-107 (part. p. 91).

6. Ibid., p. 91-92.

7. Toutes les données de cette intervention sur l'activité de l'IICCR ont été obtenues à partir de son site officiel, http://www.crimelecomunismului.ro, consulté le 29.10.2007 et le 19.11.2007.

8. Décision du Gouvernement $\mathrm{n}^{\circ} 1724,21$ décembre 2005 (publiée dans le Journal Officiel, $\mathrm{n}^{\circ}$ 1195, 30 décembre 2005).

9. Petre Mihai Băcanu, Elvira Ruxandra Simona ştefana Bianu, Doina Cornea, Dennis Deletant, Constantin Ticu-Dumitrescu, Radu Filipescu, Radu Ioanid, Gabriel Liiceanu, Marius Oprea, Cristian Pârvulescu et Andrei Pleşu. 
10. Marius Oprea (Président), Stejărel Olaru (Directeur général) et Lucia Hossu-Longin (Secrétaire général).

11. Décision du Gouvernement, $\mathrm{n}^{\circ} 388,25$ avril 2007 (publiée dans Le Journal Officiel, $\mathrm{n}^{\circ} 295$, 04 mai 2007).

12. Voir à cet égard les données des fiches suivantes, disponibles sur le site officiel de l'institution: "L'identification des anciens commandants et adjoints des pénitentiaires "; "Le rapport sur les recherches archéologiques faites dans le cimetière des pauvres de Sighetul MarmaŢiei les 2/4 novembre 2006 "; «La campagne d'investigation de Salcia - Insula Mare a Brăilei, les 27/29 mars 2007 », etc.

13. De ce trebuie condamnat comunismul, Anuarul IICCR [Pourquoi faut-il condamner le communisme? L'Annuaire de l'IICCR], I, 2006; voir aussi l'Annuaire de 2007 (Élite communiste înainte şi după 1989 [ Élites communistes avant et après 1989].

14. Lucien Febvre, Combats pour l'histoire, Paris, Gallimard, 1933. Jacques Le Goff, Histoire et Mémoire, Paris, Gallimard, 1988.

15. BBCRomanian.com, 24 octobre 2007, consulté le 29 octobre 2007.

16. Ibid.

\section{AUTEUR}

\section{LAURENȚIU VLAD}

Université de Bucarest 